Retraction

\title{
Retracted: Genetic and Functional Profiling of Crohn's Disease: Autophagy Mechanism and Susceptibility to Infectious Diseases
}

\author{
BioMed Research International \\ Received 7 October 2013; Accepted 7 October 2013 \\ Copyright (C) 2013 BioMed Research International. This is an open access article distributed under the Creative Commons \\ Attribution License, which permits unrestricted use, distribution, and reproduction in any medium, provided the original work is \\ properly cited.
}

This article has been retracted as it is found to contain a substantial amount of material from published papers. The three most plagiarized papers are: (1) I. Sekirov, S. L. Russell, L. C. Antunes and B. B. Finlay, "Gut microbiota in health and disease," Physiological Reviews, vol. 90, no. 3, pp. 859-904, 2010. (2) V. M. Hubbard and K. Cadwell, "Viruses, autophagy genes, and Crohn's disease," Viruses, vol. 3, no. 7, pp. 1281-1311, 2011. (3) B. Khor, A. Gardet and R. J. Xavier, "Genetics and pathogenesis of inflammatory bowel disease," Nature, vol. 474, no. 7351, pp. 307-317, 2011 [1].

\section{References}

[1] A. Marcuzzi, A. M. Bianco, M. Girardelli et al., "Genetic and functional profiling of Crohn's disease: autophagy mechanism and susceptibility to infectious diseases," BioMed Research International, vol. 2013, Article ID 297501, 11 pages, 2013. 


\title{
Genetic and Functional Profiling of Crohn's Disease: Autophagy Mechanism and Susceptibility to Infectious Diseases
}

\author{
Annalisa Marcuzzi, ${ }^{1}$ Anna Monica Bianco, ${ }^{1}$ Martina Girardelli, ${ }^{1}$ Alberto Tommasini, \\ Stefano Martelossi, ${ }^{1}$ Lorenzo Monasta, ${ }^{1}$ and Sergio Crovella ${ }^{1,2}$ \\ ${ }^{1}$ Institute for Maternal and Child Health-IRCCS "Burlo Garofolo" of Trieste, Via dell'Istria 65/1, 34137 Trieste, Italy \\ ${ }^{2}$ University of Trieste, 34127 Trieste, Italy
}

Correspondence should be addressed to Annalisa Marcuzzi; marcuzzi@burlo.trieste.it

Received 27 February 2013; Accepted 20 March 2013

Academic Editor: Enrique Medina-Acosta

Copyright (C) 2013 Annalisa Marcuzzi et al. This is an open access article distributed under the Creative Commons Attribution License, which permits unrestricted use, distribution, and reproduction in any medium, provided the original work is properly cited.

Crohn's disease is a complex disease in which genome, microbiome, and environment interact to produce the immunological background of the disease. Disease in childhood is more extensive and characterized by a rapid progression, leading to severe repercussions in the course of the disorder. Several genetic variations have been associated with an increased risk of developing the disease and most of these are also implicated in other autoimmune disorders. The gut has many tiers of defense against incursion by luminal microbes, including the epithelial barrier and the innate and adaptive immune responses. Moreover, recent evidence shows that bacterial and viral infections, as well as inflammasome genes and genes involved in the autophagy process, are implicated in Crohn's disease pathogenesis. The aim of this review is to establish how much the diagnostic system can improve, thus increasing the success of Crohn's disease diagnosis. The major expectation for the near future is to be able to anticipate the possible consequences of the disease already in childhood, thus preventing associated complications, and to choose the best treatment for each patient.

\section{Introduction}

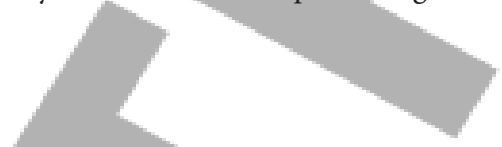

Crohn's disease $(\mathrm{CD})$ is a chronic form of inflammatory bowel disease (IBD) that can affect any part of the gastrointestinal tract, from the mouth to the anus. However, it most commonly affects the colon and terminal ileum [1] with up to $75 \%$ of patients having ileal disease with or without colonic involvement [2]. It is a debilitating disorder with an overall prevalence of $0.5 \%-1 \%$ of the general population $[3,4]$. CD differs from other types of IBDs because in patients with $C D$ the inflammation is often continuous and with involvement of the mucosa [5].

Complications are common but not a constant: disease progression is marked by severe colitis, strictures and perianal fistulas, typically requiring surgery $[6,7]$.

Beaugerie et al. [8] recently reported three factors that at the time of the diagnosis increase the chance of developing a disabling disease in the following five years: (A) age $<40$ years, (B) presence of perianal lesions, and (C) the requirement of steroids to control the first flare [8]. However, as the median age at diagnosis is 27 years, patients may live with $\mathrm{CD}$ for more than 50 years in the Western world, where life expectancy of patients exceeds 70 years.

The age of onset is frequently in the second decade of life, and most patients progress to a relapsing disease characterized by abdominal pain, bloody diarrhea, vomit, and weight loss.

Although CD normally manifests in adulthood, it can be present in childhood before the age of 2 years [9]. The early onset Crohn's disease (EOCD) is typically more extensive (beyond the colon and/or oral or perianal disease) and characterized by rapid progression, leading to severe repercussions in disease development [10]. Diagnosis is particularly challenging in children in which presenting symptoms may vary widely and may only consist of subtle extraintestinal manifestations [11]. This often leads to a typical delay in the diagnosis of pediatric IBD, ranging from 4 weeks in severe colitis to 6-7 months in milder disease. Reducing 


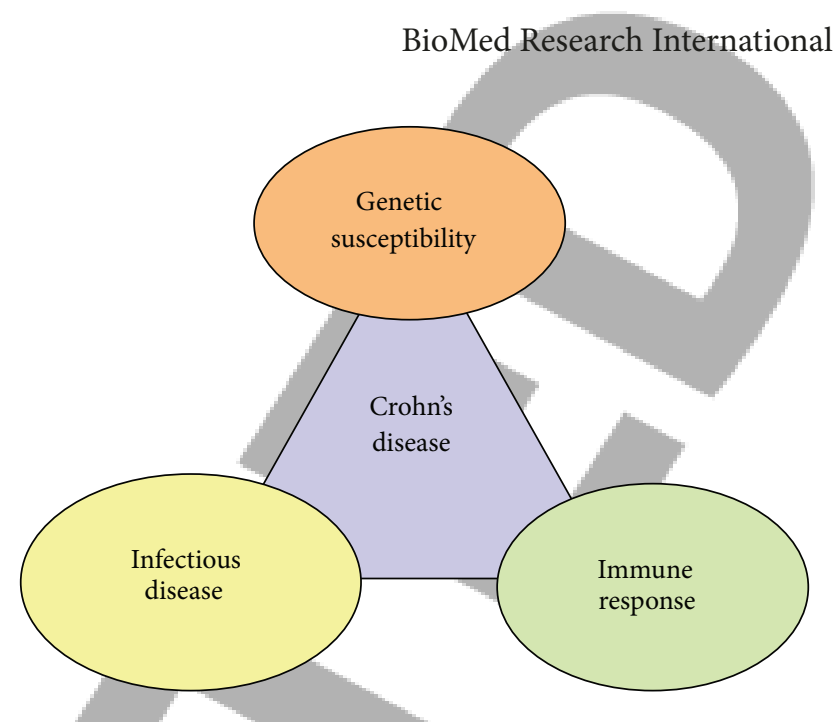

FIGURE 1: Crohn's disease is a heterogeneous disorder of multifactorial etiology in which genetic, environmental, and microbial factors, together with the immunological response, interact to produce the disease. genetic environmental and microbial factors. Given the cult genotype-phenotype correlation and given the heterogeneous genetics, the different interactions among predisposing factors, and not only the number of genes involved, should be considered in order to understand the mechanisms of this disease. Many previous and ongoing studies have sought to identify genes and especially disease-causing variants such as risk factors for the disease. Identification and characterization of disease-causing variants represents one of the biggest challenges of genetics within the etiopathogenetic study of CD. Genomewide association studies already identified several distinct genetic polymorphisms associated with Crohn's disease. In European individuals, NOD2 gene polymorphisms confer by far the greatest risk for the disease. Other variations in ATG16L1, IRGM, and IL23R genes were reported to be highly associated with CD.

The genetic background has certainly a predisposing role, but several alternative explanations are possible, mostly related to lifestyle. The importance of environmental factors is shown by an increase in the incidence rate of diseases in ethnic groups previously less affected, as Hispanic, Asians, and immigrants that had moved from regions of low incidence into areas where the incidence of the disease is higher [16].

Observation of Crohn's patients and animal models suggests a role of bacteria in the disorder [17]. Among the most important bacteria that can adhere and invade the mucosa is Escherichia coli [18]. The onset of the disease is quite common after gastrointestinal infections and people suffering from this disorder have, generally, higher concentrations of mucosal bacteria if compared to healthy subjects [19].

A detailed picture of how genes work together and interact with environmental and microbial factors may better explain individual differences in CD susceptibility.

\section{Etiology of CD}

The complex pathophysiology of CD has, for a long time, been an enigma [20].

Although the precise etiology of CD remains elusive, epidemiological data conclusively indicate a deregulation of the immune response against the luminal flora in a genetically susceptible host [21] (Figure 1).

It is commonly assumed that $\mathrm{CD}$ is a heterogeneous disorder of multifactorial etiology in which genome, microbiome (hereditability), and environment interact to produce the immunological background of the disease. It is probable that patients have a genetic predisposition for the development of the disease coupled with immunoregulation disturbances [22].

2.1. Genetic Susceptibility: Greater Weighing Factor in Early Onset Crohn Disease. The epidemiologic evidence of the role of genetic factors in the pathogenesis of the disease came from studies demonstrating higher rates of $\mathrm{CD}$ among individuals of Caucasian and Jewish ethnicity, familial aggregation of CD, and higher concordance rates of both twins developing CD in monozygotic compared to dizygotic twins. Due to the complexity of the disease, the search for specific CD susceptibility genes has been very difficult so far. Despite the large number of genomewide associations (GWAS) established to date, most complex diseases (not monogenic) have only managed to explain some additional percentage of the hereditability estimates. The source of this missing hereditability is the subject of much debate with various explanations: overestimates of original heritability statistics, underpowered GWAS studies to detect common variants, poorly investigated epistasis and gene-environment interactions, and rare genetic variants [23]. In the attempt to explain some of this missing hereditability, researchers have adopted several complementary strategies. Combined genotypes, "private genes," and epigenetic markers may account for this missing hereditability: monogenic immunity disorders are increasingly diagnosed in patients with EOCD [24]. Advances in bioinformatics have now made it possible to perform GWAS using copy number variation probes. By several GWAS and meta-analysis studies many genes have been associated with CD: more than 90 distinct genomic loci have been found to be associated with an increased risk of 

(1) 


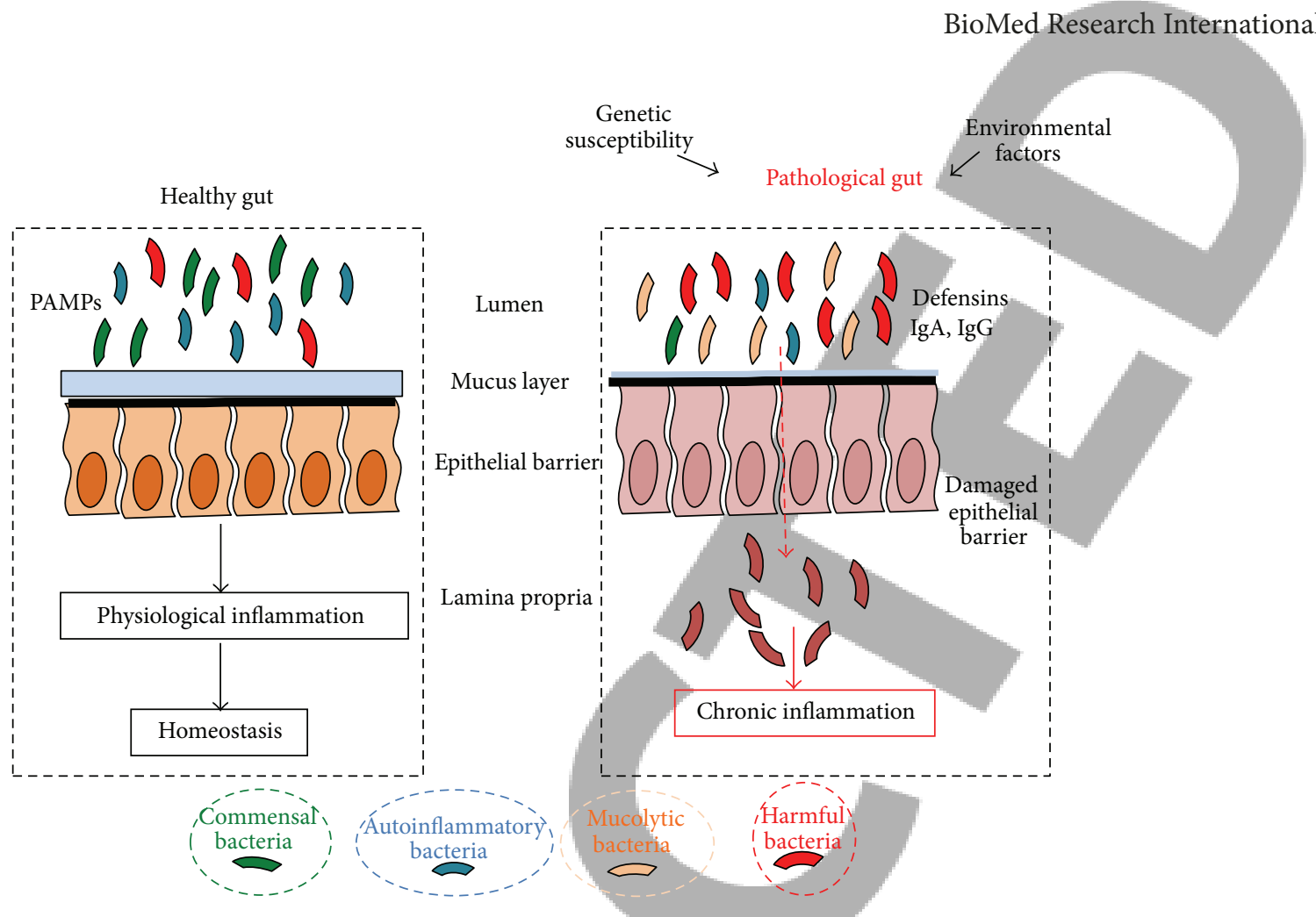

FIgURE 3: The role of microbiota in Crohn's disease pathogenesis. The interplay between the host microbiota and the environmental factors in a genetic susceptible host results in a progressive inflammatory damage to the host intestinal mucosa.

for microbial colonization. It is estimated that the human microbiota, not homogeneous in the GIT, contains as many as 1014 bacterial cells $[40,41]$ and the number of bacterial species present in the human gut is estimated to be 500 to 1000 [42]. Nevertheless, a recent analysis involving multiple subjects has suggested that the collective human gut microbiota is composed of over 35000 bacterial species [43].

Colonization of the human gut with microbes begins immediately at birth; in fact infants are exposed to a complex microbial population upon the passage through the birth canal [44] and it is known that infants delivered through cesarean section have different microbial compositions compared to vaginally delivered infants [45]. It has been shown that the microbiota of adult monozygotic and dizygotic twins was equally similar to that of their siblings, suggesting that the colonization by the microbiota from a shared mother was more decisive in determining their adult microbiota than their genetic makeup [46] (Figure 3).

Several studies have shown that host genetics can impact the microbial composition of the gut $[47,48]$. The central role of gut microbiota in the development of mucosal immunity is not surprising considering that the intestinal mucosa represents the largest surface area in contact with the antigens of the external environment called PAMPs (pathogenassociated molecular patterns). Additionally, the dense carpet of the gut microbiota overlying the mucosa normally accounts for the largest proportion of the antigens presented to the resident immune cells and those stimulating the pattern recognition receptors such as the NOD-like receptors (NLRs) of the intestinal epithelial cells [49]. The GI (gastrointestinal gut) microbiome of healthy humans is dominated by four major bacterial phyla: Firmicutes, Bacteroidetes, and to a lesser degree Proteobacteria and Actinobacteria [50]. Many studies have observed imbalances or dysbioses in the GI microbiomes of $C D$ patients $[51,52]$. In CD patients biodiversity is decreased, with a lower proportion of Firmicutes and an increase in Gammaproteobacteria [53]. In CD, proportions of the Clostridia are altered: the Roseburia and Faecalibacterium genera of the Lachnospiraceae and Ruminococcaceae families are decreased, whereas Ruminococcus gnavus increases [54]. An important concept in the pathogenesis of CD is that bacterial and viral interactions occur in a host gene-specific manner $[55,56]$. Surgical diversion of the fecal stream ameliorates inflammation. In addition, the evaluation of the microbial populations in surgically resected tissue samples of small bowel and colon from CD patients and non-CD controls, by rRNA sequence analysis, showed that specific flora was not enriched in small bowel or colon from CD patients. However, a subset of CD samples showed alterations in the representations of the Bacteroides and Firmicutes $[43,57]$. Moreover, several studies have shown that the gut microbiota is altered in IBD patients. For example, biopsy samples from CD patients were used to prepare bacterial DNA which was amplified using universal bacterial $16 \mathrm{~S}$ rRNA primers [58], and a significant increase in Proteobacteria and Bacteroidetes was found in CD patients compared to controls, with a decrease in Clostridia. Metagenomic approaches were used to analyze fecal samples from Crohn's patients and healthy donors and revealed reduced complexity of the Firmicutes in affected individuals [59]. Finally, the evidence that intestinal bacteria play an important role in CD patients is that antibiotics help some patients and can ameliorate 
FIGURE 4: Up- and Downregulation of the proinflammatory cytokines evidenced in the immune system dysregulation of CD patients.

disease activity. Moreover metronidazole is an important therapeutic agent for certain complications of CD such as fistulising disease. Viral infection is required to generate the Paneth cell defect found in ATG16L1 mice [60], suggesting that in addition to human bacterial microbiota, viral or fungal commensals may play a role in CD pathogenesis.

\subsection{Immunological Response in $C D$}

2.3.1. Th1 and Th17 Implicated in CD Pathogenesis. Although the exact CD etiology is still not completely understood, several studies indicate that its pathogenesis is characterized by an exaggerated immune response in genetically susceptible individuals.

CD patients suffer from marked immune system deregulation. The inflammation seen in these patients is characterized by pronounced Th1 and Th17 responses [61] involving upregulation of proinflammatory cytokines IL-1, IL-6, IL-12, TNF- $\alpha$, IFN- $\gamma$, IL-23, and IL-17 and downregulation of IL- 10, but it is not clear whether this is a cause or a consequence of the disease [62] (Figure 4).

Th1 cells are commonly assumed to be associated with CD development and produce IFN- $\gamma$, and their primary role is the protection against intracellular microbes. IFN- $\gamma$ secreting lamina propria lymphocytes are abundant in the mucosa of CD patients: this condition is marked at CD onset (mucosal $\mathrm{T}$ cells appear to mount a typical Th1 response that resembles an acute infectious process) and disappears in late CD.

Recently, several studies showed the pivotal role of the imbalance of regulatory T cells (Treg) and Th17 in CD. Treg cells are important for the control of the immune response to self-antigens preventing autoimmunity and maintaining selftolerance [63]. In contrast, IL-17 producing Th17 cells were recognized as a novel group of $\mathrm{T}$ cells which play a major role in autoimmunity. The gastrointestinal immune system has to maintain both a state of tolerance toward intestinal antigens and the ability to combat pathogens. In CD this balance is lost and the effects of proinflammatory $\mathrm{T}$ cells outnumber the tolerizing, anti-inflammatory effects of Treg cells. The discovery that Th17 cells, which express the IL23 receptor (IL-23R), play a role in CD pathogenesis was supported by recent GWAS studies demonstrating that IL23R and other genes involved in the differentiation of Th17 cells are susceptibility genes.

To confirm the link between immune response and genetic susceptibility in the pathogenesis of CD there are several recent lines of evidence that the key role is played by autophagy that includes the antigen presentation and the production of proinflammatory cytokines. The relationship between autophagy and microbes, indeed, has remained illdefined until a recent convergence of studies showing that autophagy is an innate immune defense against bacteria, protozoa, and viral pathogens [64]. It is commonly assumed that the role of autophagy in addition to eliminating intracellular pathogens [65] contributes to MHC II restricted endogenous antigen presentation. It is an effector of Th1/Th2 polarization, affects $\mathrm{B}$ and $\mathrm{T}$ cell homeostasis and repertoire selection, delivers cytosolic PAMP or danger associated molecular patterns to endosomal toll-like receptors (TLR), and acts as an innate immunity effector downstream of TLR [66]. Polymorphisms in autophagy genes result in deregulation of these processes and affect gut homeostasis: genetic variants of autophagy genes have been linked to CD.

2.3.2. The Role of the NLRP3 Inflammasome in the Pathogenesis of $C D$. Inflammasomes are cytoplasmic multiprotein complexes that function as sensors of endogenous or exogenous PAMPs. They are composed of one of several nucleotide-binding oligomerization-domain protein-like receptors (NLRs), including NLRP1, NLRP3, NLRP6, and NLRPC4. Upon sensing the relevant signal, they assemble, typically together with an adaptor protein, an apoptosisassociated speck-like protein (ASC) or a caspase activating and recruitment domain 8 (CARD8), into a multiprotein complex that governs caspase- 1 activation and subsequent cleavage of effector proinflammatory cytokines including pro-IL-1 $\beta$ and pro-IL-18. 
Recently several studies highlighted with particular emphasis the relevance and the role of NLRP3 (previously known as CIAS1 and NLRP3) in the pathogenesis of CD $[67,68]$.

There is evidence suggesting that NLRP3 is able to respond to a variety of signals: adenosine triphosphate (ATP), nigericin, maitotoxin, Staphylococcus aureus and Listeria monocytogenes [69], and RNA and uric acid crystals (monosodium urate and calcium pyrophosphate dehydrate) released from dying cells $[70,71]$.

Literature data showed that the proinflammatory compound muramyl-dipeptide, the minimal bioactive peptidoglycan motif common to all bacteria, was an activator of the NLRP3 inflammasome, which suggested a very interesting connection between NOD2 and NALP3 [72].

On the other hand, Kanneganti et al. suggested that bacterial RNA and small antiviral compounds are the specific ligands of NLRP3 rather than MDP [70].

Anyway, NLRP3 plays a pivotal role in the inflammation regulating the activation of the caspase- 1 and processing of IL- $1 \beta$, two key mediators involved in the pathogenesis of the more common inflammatory disorders [73].

More recently, in an increasingly complicated picture, Elinav et al. described a novel regulatory sensing system in the colon, dependent on the NLRP6 inflammasome [74], and von Kampen et al. showed that CARD8 negatively regulates NOD-2 mediated signaling [75]. These current data further underline the link between the different components of the $\mathrm{CD}$ etiopathogenesis that are strongly correlated.

\section{Autophagy in CD}

Genomewide association studies and genetic analyses have emphasized the involvement of autophagy processes in the pathogenesis of inflammatory bowel diseases implicating three component genes in CD pathogenesis: ATG16L1 [76], IRGM [77], and NOD2 [78, 79]. These genes encode proteins critical for autophagy, a process that mediates degradation of intracellular proteins via vesicle-mediated delivery to the lysosome [80, 81]. Autophagy is involved in intracellular homeostasis, contributing to the degradation and recycling of cytosolic contents and organelles, as well as to resistance against infection and the removal of intracellular microbes. It is a major degradative pathway of the cell with several critical functions in innate and adaptive immunity [82] (Figure 5).

The ATG16L1 deficiency mouse showed Paneth cell dysfunction with aberrant exocytosis, as well as an altered transcriptional profile, characterized by increased expression of pro-inflammatory cytokines and lipid metabolism genes like Paneth cell phenotype of CD patients [83]. Nevertheless an important observation derived from the ATG16L1 mouse model was that the murine norovirus infection, as well as the presence of the commensal bacteria, was required for the generation of these specific Paneth cell abnormalities [84]. ATG16L1 is essential for all forms of autophagy, and the coding mutation T300A is associated with increased risk of CD. Despite its ubiquitous expression, the defects associated with ATG16L1 polymorphisms have so far been described only within the gut, probably owing to the high microbial load in this tissue.

Nucleotide-binding-oligomerization-domain- (NOD-) like receptors (NLRs) represent ancient sentinels of the host innate immune system, and genetic variants in NLR genes are associated with complex chronic inflammatory barrier diseases [85]. The NOD2 gene is an intracellular sensor for the bacterial cell wall component muramyl-dipeptide, and loss-of-function variants in the human NOD2 gene have been associated with an increased susceptibility for CD $[86,87]$ in Caucasian populations of European ancestry [88], and particularly for ileal disease [89], and were found to be an important regulator of the commensal gut microbiota in mice [90]. NOD2 recognizes components of the bacterial cell wall and elicits an NF- $\kappa \mathrm{B}$ response and mediates the release of defensins, which are antimicrobial peptides. Evidence from MDP stimulation of NOD2-activated autophagy shows a link between genetic risk loci and highlights the importance of defining disease associated pathways and the potential of new roles for known genes [78]. Epithelial cells and dendritic cells containing Crohn's-disease-associated ATG16L1 and NOD2 variants show defects in antibacterial autophagy $[79,91]$. In dendritic cells, these defects are associated with an impaired ability to present exogenous antigens to CD4+T cells [78]. A discussed model of Crohn's disease is the one in which individuals are genetically susceptible to a pathogen that triggers a compensatory and harmful immune response. Antibacterial autophagy, through ATG16L1, NOD2, and potentially other genes (IRGM), is consistent with this model. However, one of the most important experimental supports for this model comes from an unrelated study using Citrobacter rodentium to induce intestinal inflammation in NOD2 -/- mice [92]. These results illustrate a close relationship between NOD2, ATG16L1, and autophagy, affecting intracellular processing and communication with the adaptive immune system suggesting that genetic polymorphisms may affect both pathways concomitantly.

IRGM belongs to the p47 Immunity-Related GTPase (IRG) family and is linked to CD by GWAS as a protein that is implicated in the autophagy mechanism [93]. The analysis of the interactions between 44 autophagy-associated human proteins and 83 viral proteins belonging to different RNA virus families revealed that IRGM was the autophagyassociated protein most targeted by these viruses. IRGM can interact with 12 viral proteins belonging to different viruses, such as HCV and HIV-1 [94, 95]. A recent study suggests that a polymorphism in IRGM could affect the binding and the consequent misregulation by a specific miRNA (miR-196) that is highly expressed in the intestinal tissue of patients with Crohn's disease. The consequence is that the xenophagy flux is not well regulated leading to the accumulation of bacteria in the lysosomal compartment. This study showed that greater IRGM expression leads to both colocalization of adherent invasive E. coli (AIEC) with the autophagy machinery and increased intracellular survival of the bacteria [96]. This and other strains of $E$. coli are more abundant in the mucosa of CD patients [90]. The ability of IRGM to induce autophagy and limit the replication of intracellular bacteria has been demonstrated with mycobacteria by inducing mitochondrial 


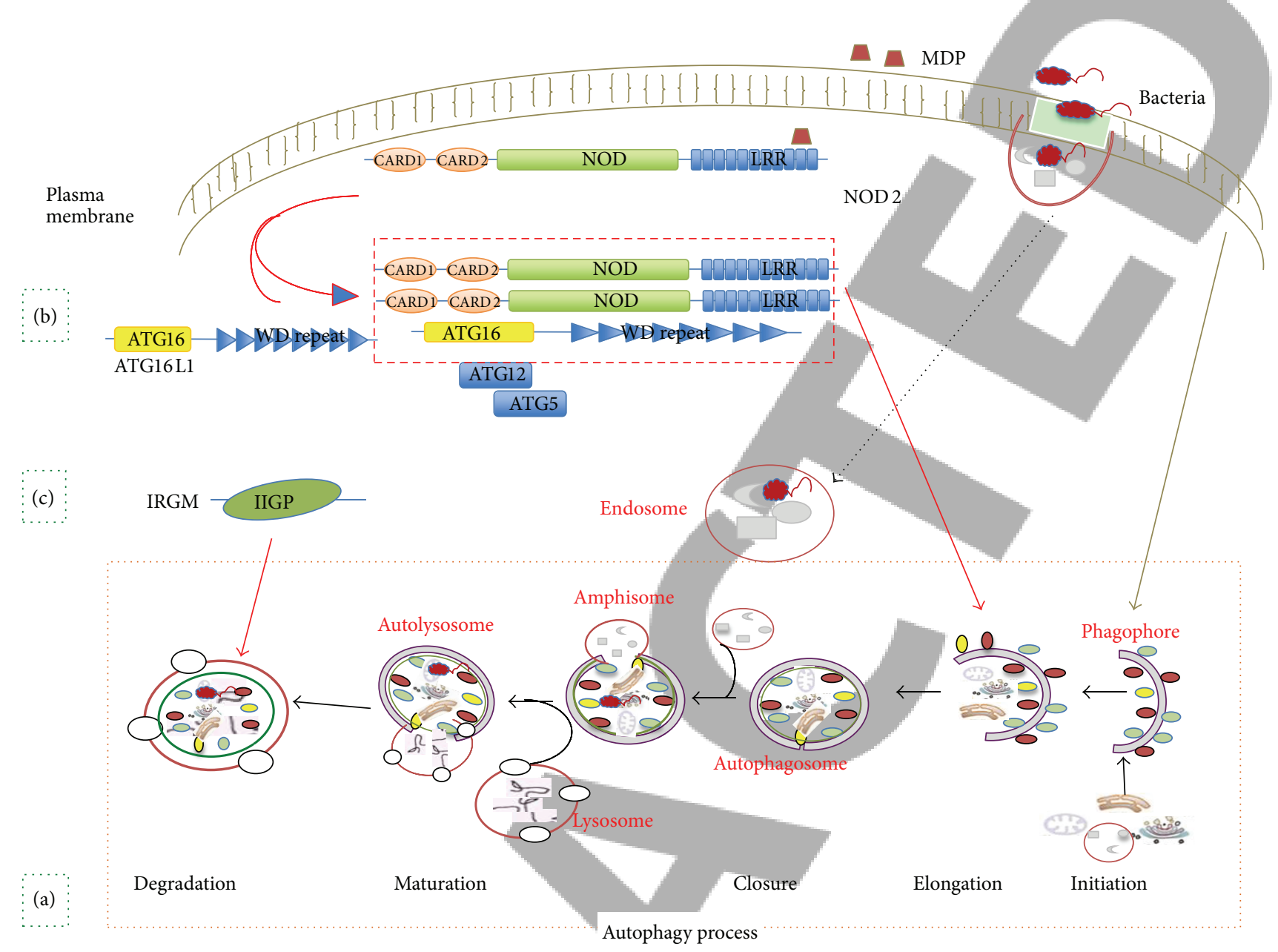

FIGURE 5: CD pathogenesis and autophagy: susceptible CD genes ATG16L1, NOD2, and IRGM are proteins critical for the autophagy process. (a) The process of mammalian autophagy is divided into the following principal steps: initiation, elongation, closure, maturation, and degradation. (b) At the bacterial entry site NOD2 activated by MDP recruit ATG16L1 to the plasma membrane. Follow the assembling of the ATG5-ATG12 complex, stabilized by ATG16L1, that facilitates the formation of an autophagosome around the invading bacterium. (c) IRGM, another autophagy-related gene, could be involved in the final steps of the degradation step.

depolarization and can increase ROS production and cell death [97]. Finally IRGM could regulate inflammation by either regulating intracellular pathogens or cellular homeostasis much like ATG16L1.

These data provide further information and support for the hypothesis that microbial/viral interactions with the intestinal mucosa are required for disease generation and suggest that combinatorial models for $\mathrm{CD}$ pathogenesis are most relevant for the study of human disease pathogenesis.

\section{Concluding Remarks}

The diagnosis of $\mathrm{CD}$ is reached through the results of clinical, laboratory, radiographic, endoscopic, and histologic analyses.

Radiological and endoscopic techniques are essential for the diagnosis of CD since its onset and are useful in assessing the inflammatory status of the intestinal mucosa. However, endoscopy is an invasive procedure. In children it can be traumatic and could have critical implications due to the more severe clinical manifestation and complication of the pediatric disease, that make the intestinal mucosal extremely thin and at risk of perforation.

Noninvasive tests for $\mathrm{CD}$ already exist, including antibodies, imaging-based screens, and fecal biomarkers [98]. The specificity of existing methods ranges from $89 \%$ to $95 \%$ for $\mathrm{CD}$ and other inflammatory bowel diseases. However, these methods are limited to active disease and poorly sensitive ( $55 \%)$. Their outcome can be confounded by other diseases, further limiting their clinical utility. Recently, high expectations are placed in diagnostic studies of the gastrointestinal microbiota, but further validations will be necessary before this tool is accepted in clinical practice [32, 99].

Research is moving forward in order to identify new and valid biomarkers for the diagnosis of the disease with the aim of replacing the use of invasive techniques.

Currently, only the measurement of fecal calprotectin levels has achieved a place in clinical routine practice and is used as a marker for noninvasive determination of intestinal 
inflammation $[99,100]$. This protein is an ideal marker because it is not degraded by the human microbiota. Levels of fecal calprotectin significantly increase in patients with CD, ulcerative colitis, infectious colitis, and, to a lesser extent, in tumors of the colon rectum, but not in patients with functional disorders, as in the case of the irritable bowel syndrome. In $\mathrm{CD}$, the calprotectin assay reflects the activity of the disease, monitoring its progression, and can contribute to the decision about the correct medication strategy.

The application of this test in the pediatric population is a good result. Although the test is not yet able to replace diagnostic colonoscopy, it can be a good indicator for the decision to use or delay the use of invasive investigations [99].

Recently, Vitali et al. suggested the use of high-mobility group boxl (HMGB1) as a novel marker of intestinal mucosal inflammation. HMGB1 is today regarded as a pleiotropic cytokine, that is passively released by necrotic cells, but not from apoptotic cells. Moreover HMGB1 could be actively secreted from some types of immune cells in response to lipopolysaccharide (LPS), IFN- $\gamma$, and TNF- $\alpha$. There is evidence that HMGB1 is secreted in the stools of these patients and not detectable in controls [101].

The relationship between the genetic susceptibility and the microbiome could be considered in the disease diagnosis. There are several international human microbiome projects that have focused initially on the bacterial component of the microbiome. The evidence that bacteria play an important role in $\mathrm{CD}$ includes the observation that surgical diversion of the fecal stream ameliorates the inflammation and that antibiotics help some patients. Moreover other evidence is showed in mouse models of colitis where virus, bacteria, or both acting together can contribute to the pathology via signaling through innate immune sensors and regulation of pro- and anti-inflammatory cytokines [82]. The microbiome varies from person to person and such variation could provide environmental inputs that contribute to the incidence of CD, within the genetic foundation revealed by GWAS [102]. The concept of dysbiosis as a contributor to CD is correlated with intestinal bacteria communities, so the changes in the bacterial microbiota could have a potential role in the disease. However, this hypothesis needs to be expanded to include specific interactions between individual bacteria and host genes.

Moreover, another ambitious goal is the identification of a genetic pattern able to associate specific phenotypic characteristics to $\mathrm{CD}$ patients or to anticipate the possible consequences of the disease already in childhood and thus prevent complications associated with the disease and to choose the best treatment for each patient.

A rapid diagnosis is fundamental to avoid a growth delay or complications of the disease typical of the pediatric disease leading to surgery. In some cases, genetic studies have provided useful information for the identification of specific mutations that predict risk of stenosis and surgery and/or disease localization in pediatric-onset CD $[103,104]$.

The difficulty of finding a common genetic pattern of association is caused by the multifactorial feature of the disease that shows different characterizations by world region and race.
In conclusion, it would certainly be useful to be able to create a biological algorithm that helps clinicians in the identification and classification of the disease and to determine the pharmacological care. This algorithm could include not only the known and principal factors predisposing to the disease, but also the gene-microbiome interaction and could help identify novel markers in patients with familiar history of EOCD. This could represent a major advance for early-onset diagnosis as specific tests might be developed to improve counselling, while direct identification of modifier genes might assist in the recognition of new genetic, environmental, and microbial causes of CD.

\section{Conflict of Interests}

The authors declare they have no conflict of interests related to the issues discussed in this paper.

\section{Authors' Contribution}

Annalisa Marcuzzi and Anna Monica Bianco contributed equally to this study.

\section{References}

[1] D. C. Baumgart and W. J. Sandborn, "Crohn's disease," The Lancet, vol. 380, no. 9853, pp. 1590-1605, 2012.

[2] G. Radford-Smith and N. Pandeya, "Associations between NOD2/CARD15 genotype and phenotype in Crohn's diseaseare we there yet?" World Journal of Gastroenterology, vol. 12, no. 44, pp. 7097-7103, 2006.

[3] D. K. Bonen and J. H. Cho, "The genetics of inflammatory bowel disease," Gastroenterology, vol. 124, no. 2, pp. 521-536, 2003.

[4] S. K. Yang, S. Yun, J. H. Kim et al., "Epidemiology of inflammatory bowel disease in the Songpa-Kangdong district, Seoul, Korea, 1986-2005: a KASID study," Inflammatory Bowel Diseases, vol. 14, no. 4, pp. 542-549, 2008.

[5] R. J. Xavier and D. K. Podolsky, "Unravelling the pathogenesis of inflammatory bowel disease," Nature, vol. 448, no. 7152, pp. 427-434, 2007.

[6] J. Cosnes, S. Cattan, A. Blain et al., "Long-term evolution of disease behavior of Crohn's disease," Inflammatory Bowel Diseases, vol. 8, no. 4, pp. 244-250, 2002.

[7] E. Louis, A. Collard, A. F. Oger, E. Degroote, F. A. Aboul Nasr El Yafi, and J. Belaiche, "Behaviour of Crohn's disease according to the Vienna classification: changing pattern over the course of the disease," Gut, vol. 49, no. 6, pp. 777-782, 2001.

[8] L. Beaugerie, P. Seksik, I. Nion-Larmurier, J. P. Gendre, and J. Cosnes, "Predictors of Crohn's disease," Gastroenterology, vol. 130, no. 3, pp. 650-656, 2006.

[9] M. B. Heyman, B. S. Kirschner, B. D. Gold et al., "Children with early-onset inflammatory bowel disease (IBD): analysis of a pediatric IBD consortium registry," Journal of Pediatrics, vol. 146, no. 1, pp. 35-40, 2005.

[10] Z. Cannioto, I. Berti, S. Martelossi et al., "IBD and IBD mimicking enterocolitis in children younger than 2 years of age," European Journal of Pediatrics, vol. 168, no. 2, pp. 149-155, 2009.

[11] IBD Working Group of the European Society for Paediatric Gastroenterology, Hepatology and Nutrition, "Inflammatory 
bowel disease in children and adolescents: recommendations for diagnosis-the Porto criteria," Journal of Pediatric Gastroenterology and Nutrition, vol. 41, no. 1, pp. 1-7, 2005.

[12] C. Spray, G. D. Debelle, and M. S. Murphy, "Current diagnosis, management and morbidity in paediatric inflammatory bowel disease," Acta Paediatrica, vol. 90, no. 4, pp. 400-405, 2001.

[13] J. D. Lewis, O. Abramson, M. Pascua et al., "Timing of myelosuppression during thiopurine therapy for inflammatory bowel disease: implications for monitoring recommendations," Clinical Gastroenterology and Hepatology, vol. 7, no. 11, pp. 11951201, 2009.

[14] K. Mitsuyama, N. Tomiyasu, K. Takaki et al., "Interleukin-10 in the pathophysiology of inflammatory bowel disease: increased serum concentrations during the recovery phase," Mediators of Inflammation, vol. 2006, Article ID 26875, 7 pages, 2006.

[15] S. J. Bickston, L. W. Comerford, and F. Cominelli, "Future therapies for inflammatory bowel disease," Current Gastroenterology Reports, vol. 5, no. 6, pp. 518-523, 2003.

[16] J. K. Hou, H. El-Serag, and S. Thirumurthi, "Distribution and manifestations of inflammatory bowel disease in Asians, Hispanics, and African Americans: a systematic review," American Journal of Gastroenterology, vol. 104, no. 8, pp. 2100-2109, 2009.

[17] C. Abraham and J. H. Cho, "Inflammatory bowel disease," The New England Journal of Medicine, vol. 361, no. 21, pp. 20662078, 2009.

[18] N. Barnich, F. A. Carvalho, A. L. Glasser et al., "CEACAM6 acts as a receptor for adherent-invasive E. coli, supporting ileal mucosa colonization in Crohn disease," The Journal of Clinical Investigation, vol. 117, no. 6, pp. 1566-1574, 2007.

[19] D. C. Baumgart and W. J. Sandborn, "Inflammatory bowel disease: clinical aspects and established and evolving therapies," The Lancet, vol. 369, no. 9573, pp. 1641-1657, 2007.

[20] D. K. Podolsky, "The current future understanding of inflammatory bowel disease," Best Practice \& Research Clinical Gastroenterology, vol. 16, no. 6, pp. 933-943, 2002.

[21] A. Gutierrez, M. Scharl, L. Sempere et al., "Genetic susceptibility to increased bacterial translocation influences the response to biological therapy in patients with Crohn's disease," Gut, 2013.

[22] J. P. Achkar and R. Duerr, "The expanding universe of inflammatory bowel disease genetics," Current Opinion in Gastroenterology, vol. 24, no. 4, pp. 429-434, 2008.

[23] W. Bodmer and I. Tomlinson, "Rare genetic variants and the risk of cancer," Current Opinion in Genetics \& Development, vol. 20, no. 3, pp. 262-267, 2010.

[24] D. A. van Heel, S. Ghosh, M. Butler et al., "Muramyl dipeptide and toll-like receptor sensitivity in NOD2-associated Crohn's disease," The Lancet, vol. 365, no. 9473, pp. 1794-1796, 2005.

[25] J. van Limbergen, D. C. Wilson, and J. Satsangi, “The genetics of Crohn's disease," Annual Review of Genomics and Human Genetics, vol. 10, pp. 89-116, 2009.

[26] C. W. Lees, J. C. Barrett, M. Parkes, and J. Satsangi, "New IBD genetics: common pathways with other diseases," Gut, vol. 60, no. 12, pp. 1739-1753, 2011.

[27] S. Haglund, S. Almer, C. Peterson, and J. Söderman, "Gene expression and thiopurine metabolite profiling in inflammatory bowel disease-novel clues to drug targets and disease mechanisms?" PLoS ONE, vol. 8, no. 2, Article ID e56989, 2013.

[28] L. A. Hindorff, P. Sethupathy, H. A. Junkins et al., "Potential etiologic and functional implications of genome-wide association loci for human diseases and traits," Proceedings of the National Academy of Sciences of the United States of America, vol. 106, no. 23, pp. 9362-9367, 2009.
[29] L. D. Notarangelo, "Primary immunodeficiencies," The Journal of Allergy and Clinical Immunology, vol. 125, no. 2, supplement 2, pp. S182-S194, 2010.

[30] A. Tommasini, A. Pirrone, G. Palla et al., "The universe of immune deficiencies in Crohn's disease: a new viewpoint for an old disease?" Scandinavian Journal of Gastroenterology, vol. 45, no. 10, pp. 1141-1149, 2010.

[31] L. de Ridder, R. K. Weersma, G. Dijkstra et al., "Genetic susceptibility has a more important role in pediatric-onset Crohn's disease than in adult-onset Crohn's disease," Inflammatory Bowel Diseases, vol.13, no. 9, pp. 1083-1092, 2007.

[32] V. Biank, U. Broeckel, and S. Kugathasan, "Pediatric inflammatory bowel disease: clinical and molecular genetics," Inflammatory Bowel Diseases, vol. 13, no. 11, pp. 1430-1438, 2007.

[33] M. Lacher, R. Kappler, S. Berkholz, H. Baurecht, D. von Schweinitz, and S. Koletzko, "Association of a CXCL9 polymorphism with pediatric Crohn's disease," Biochemical and Biophysical Research Communications, vol. 363, no. 3, pp. 701707, 2007.

[34] E. O. Glocker, D. Kotlarz, K. Boztug et al., "Inflammatory bowel disease and mutations affecting the interleukin-10 receptor," The New England Journal of Medicine, vol. 361, no. 21, pp. 2033-2045, 2009.

[35] M. Imielinski, R. N. Baldassano, A. Griffiths et al., "Common variants at five new loci associated with early-onset inflammatory bowel disease," Nature Genetics, vol. 41, no. 12, pp. 13351340, 2009.

[36] D. K. Amre, D. R. Mack, K. Morgan et al., "Association between genome-wide association studies reported SNPs and pediatriconset Crohn's disease in Canadian children," Human Genetics, vol. 128, no. 2, pp. 131-135, 2010.

[37] K. L. Helbig, M. Nothnagel, J. Hampe et al., "A case-only study of gene-environment interaction between genetic susceptibility variants in NOD2 and cigarette smoking in Crohn's disease aetiology," BMC Medical Genetics, vol. 13, article 14, 2012.

[38] C. Kunz, S. Kuntz, and S. Rudloff, "Intestinal flora," Advances in Experimental Medicine and Biology, vol. 639, pp. 67-79, 2009.

[39] W. B. Whitman, D. C. Coleman, and W. J. Wiebe, "Prokaryotes: the unseen majority," Proceedings of the National Academy of Sciences of the United States of America, vol. 95, no. 12, pp. 65786583, 1998.

[40] R. E. Ley, D. A. Peterson, and J. I. Gordon, "Ecological and evolutionary forces shaping microbial diversity in the human intestine," Cell, vol. 124, no. 4, pp. 837-848, 2006.

[41] H. Verstraelen, "Cutting edge: the vaginal microflora and bacterial vaginosis," Verhandelingen-Koninklijke Academie voor Geneeskunde van België, vol. 70, no. 3, pp. 147-174, 2008.

[42] J. I. Gordon, "Honor thy gut symbionts redux," Science, vol. 336, no. 6086, pp. 1251-1253, 2012.

[43] D. N. Frank, A. L. St Amand, R. A. Feldman, E. C. Boedeker, N. Harpaz, and N. R. Pace, "Molecular-phylogenetic characterization of microbial community imbalances in human inflammatory bowel diseases," Proceedings of the National Academy of Sciences of the United States of America, vol. 104, no. 34, pp. 13780-13785, 2007.

[44] V. Redondo-Lopez, R. L. Cook, and J. D. Sobel, "Emerging role of lactobacilli in the control and maintenance of the vaginal bacterial microflora," Reviews of Infectious Diseases, vol. 12, no. 5, pp. 856-872, 1990.

[45] A. Huurre, M. Kalliomäki, S. Rautava, M. Rinne, S. Salminen, and E. Isolauri, "Mode of delivery-effects on gut microbiota 
and humoral immunity," Neonatology, vol. 93, no. 4, pp. 236240, 2008.

[46] E. G. Zoetendal, A. von Wright, T. Vilpponen-Salmela, K. BenAmor, A. D. L. Akkermans, and W. M. de Vos, "Mucosaassociated bacteria in the human gastrointestinal tract are uniformly distributed along the colon and differ from the community recovered from feces," Applied and Environmental Microbiology, vol. 68, no. 7, pp. 3401-3407, 2002.

[47] R. E. Ley, F. Bäckhed, P. Turnbaugh, C. A. Lozupone, R. D. Knight, and J. I. Gordon, "Obesity alters gut microbial ecology," Proceedings of the National Academy of Sciences of the United States of America, vol. 102, no. 31, pp. 11070-11075, 2005.

[48] C. Zhang, M. Zhang, S. Wang et al., "Interactions between gut microbiota, host genetics and diet relevant to development of metabolic syndromes in mice," The ISME Journal, vol. 4, no. 2, pp. 232-241, 2010.

[49] S. Rakoff-Nahoum and R. Medzhitov, "Innate immune recognition of the indigenous microbial flora," Mucosal Immunology, vol. 1, supplement 1, pp. S10-S14, 2008.

[50] P. B. Eckburg, E. M. Bik, C. N. Bernstein et al., "Diversity of the human intestinal microbial flora," Science, vol. 308, no. 5728, pp. 1635-1638, 2005.

[51] M. Baumgart, B. Dogan, M. Rishniw et al., "Culture independent analysis of ileal mucosa reveals a selective increase in invasive Escherichia coli of novel phylogeny relative to depletion of Clostridiales in Crohn's disease involving the ileum," The ISME Journal, vol. 1, no. 5, pp. 403-418, 2007.

[52] E. Li, C. M. Hamm, A. S. Gulati et al., "Inflammatory bowel diseases phenotype, $C$. difficile and NOD2 genotype are associated with shifts in human ileum associated microbial composition," PLoS ONE, vol. 7, no. 6, Article ID e26284, 2012.

[53] H. Sokol and P. Seksik, "The intestinal microbiota in inflammatory bowel diseases: time to connect with the host," Current Opinion in Gastroenterology, vol. 26, no. 4, pp. 327-331, 2010.

[54] M. Joossens, G. Huys, M. Cnockaert et al., "Dysbiosis of the faecal microbiota in patients with Crohn's disease and their unaffected relatives," Gut, vol. 60, no. 5, pp. 631-637, 2011.

[55] H. W. Virgin, E. J. Wherry, and R. Ahmed, "Redefining chronic viral infection," Cell, vol. 138, no. 1, pp. 30-50, 2009.

[56] E. Elinav, J. Henao-Mejia, and R. A. Flavell, "Integrative inflammasome activity in the regulation of intestinal mucosal immune responses," Mucosal Immunology, vol. 6, no. 1, pp. 4-13, 2013.

[57] D. N. Frank, C. E. Robertson, C. M. Hamm et al., "Disease phenotype and genotype are associated with shifts in intestinalassociated microbiota in inflammatory bowel diseases," Inflammatory Bowel Diseases, vol. 17, no. 1, pp. 179-184, 2011.

[58] U. Gophna, K. Sommerfeld, S. Gophna, W. F. Doolittle, and S. J. O. Veldhuyzen van Zanten, "Differences between tissueassociated intestinal microfloras of patients with Crohn's disease and ulcerative colitis," Journal of Clinical Microbiology, vol. 44, no. 11, pp. 4136-4141, 2006.

[59] C. Manichanh, L. Rigottier-Gois, E. Bonnaud et al., "Reduced diversity of faecal microbiota in Crohn's disease revealed by a metagenomic approach," Gut, vol. 55, no. 2, pp. 205-211, 2006.

[60] K. Cadwell, "Crohn's disease susceptibility gene interactions, a NOD to the newcomer ATG16L1," Gastroenterology, vol. 139, no. 5, pp. 1448-1450, 2010.

[61] D. M. Glubb, R. B. Gearry, M. L. Barclay et al., "NOD2 and ATG16L1 polymorphisms affect monocyte responses in Crohn's disease," World Journal of Gastroenterology, vol. 17, no. 23, pp. 2829-2837, 2011.
[62] J. P. Y. Ting, D. L. Kastner, and H. M. Hoffman, "CATERPILLERs, pyrin and hereditary immunological disorders," Nature Reviews Immunology, vol. 6, no. 3, pp. 183-195, 2006.

[63] S. Brand, "Crohn's disease: Th1, Th17 or both? The change of a paradigm: new immunological and genetic insights implicate Th17 cells in the pathogenesis of Crohn's disease," Gut, vol. 58, no. 8, pp. 1152-1167, 2009.

[64] T. S. Plantinga, L. A. Joosten, J. W. van der Meer, and M. G. Netea, "Modulation of inflammation by autophagy: consequences for Crohn's disease," Current Opinion in Pharmacology, vol. 12, no. 4, pp. 497-502, 2012.

[65] B. Levine and V. Deretic, "Unveiling the roles of autophagy in innate and adaptive immunity," Nature Reviews Immunology, vol. 7, no. 10, pp. 767-777, 2007.

[66] V. Deretic, "Autophagy as an innate immunity paradigm: expanding the scope and repertoire of pattern recognition receptors," Current Opinion in Immunology, vol. 24, no. 1, pp. 21-31, 2012.

[67] A. C. Villani, M. Lemire, G. Fortin et al., "Common variants in the NLRP3 region contribute to Crohn's disease susceptibility," Nature Genetics, vol. 41, no. 1, pp. 71-76, 2009.

[68] L. Ferrero-Miliani, O. H. Nielsen, P. S. Andersen, and S. E. Girardin, "Chronic inflammation: importance of NOD2 and $N A L P 3$ in interleukin-1 $\beta$ generation," Clinical and Experimental Immunology, vol. 147, no. 2, pp. 227-235, 2007.

[69] A. Gombault, L. Baron, and I. Couillin, "ATP release and purinergic signaling in NLRP3 inflammasome activation," Frontiers in Immunology, vol. 3, article 414, 2012.

[70] T. D. Kanneganti, N. Özören, M. Body-Malapel et al., "Bacterial RNA and small antiviral compounds activate caspase-1 through cryopyrin/NALP3," Nature, vol. 440, no. 7081, pp. 233-236, 2006.

[71] S. R. Kingsbury, P. G. Conaghan, and M. F. McDermott, "The role of the NLRP3 inflammasome in gout," Journal of Inflammation Research, vol. 4, no. 1, pp. 39-49, 2011.

[72] F. Martinon, L. Agostini, E. Meylan, and J. Tschopp, "Identification of bacterial muramyl dipeptide as activator of the NALP3/cryopyrin inflammasome," Current Biology, vol. 14, no. 21, pp. 1929-1934, 2004.

[73] S. Mariathasan and D. M. Monack, "Inflammasome adaptors and sensors: intracellular regulators of infection and inflammation," Nature Reviews Immunology, vol. 7, no. 1, pp. 31-40, 2007.

[74] E. Elinav, T. Strowig, A. L. Kau et al., "NLRP6 inflammasome regulates colonic microbial ecology and risk for colitis," Cell, vol. 145, no. 5, pp. 745-757, 2011.

[75] O. von Kampen, S. Lipinski, A. Till et al., "Caspase recruitment domain-containing protein 8 (CARD8) negatively regulates NOD2-mediated signaling," The Journal of Biological Chemistry, vol. 285, no. 26, pp. 19921-19926, 2010.

[76] J. Hampe, A. Franke, P. Rosenstiel et al., "A genome-wide association scan of nonsynonymous SNPs identifies a susceptibility variant for Crohn disease in ATG16L1," Nature Genetics, vol. 39, no. 2, pp. 207-211, 2007.

[77] S. A. McCarroll, A. Huett, P. Kuballa et al., "Deletion polymorphism upstream of IRGM associated with altered IRGM expression and Crohn's disease," Nature Genetics, vol. 40, no. 9, pp. 1107-1112, 2008.

[78] R. Cooney, J. Baker, O. Brain et al., "NOD2 stimulation induces autophagy in dendritic cells influencing bacterial handling and antigen presentation," Nature Medicine, vol. 16, no. 1, pp. 90-97, 2010 . 
[79] L. H. Travassos, L. A. M. Carneiro, M. Ramjeet et al., "Nod1 and NOD2 direct autophagy by recruiting ATG16L1 to the plasma membrane at the site of bacterial entry," Nature Immunology, vol. 11, no. 1, pp. 55-62, 2010.

[80] D. Glick, S. Barth, and K. F. Macleod, "Autophagy: cellular and molecular mechanisms," The Journal of Pathology, vol. 221, no. 1, pp. 3-12, 2010.

[81] A. Huett and R. J. Xavier, "Autophagy at the gut interface: mucosal responses to stress and the consequences for inflammatory bowel diseases," Inflammatory Bowel Diseases, vol. 16, no. 1, pp. 152-174, 2010.

[82] J. M. Yuk, T. Yoshimori, and E. K. Jo, "Autophagy and bacterial infectious diseases," Experimental \& Molecular Medicine, vol. 44, no. 2, pp. 99-108, 2012.

[83] C. R. Homer, A. L. Richmond, N. A. Rebert, J. Achkar, and C. McDonald, "ATG16L1 and NOD2 interact in an autophagydependent antibacterial pathway implicated in Crohn's disease pathogenesis," Gastroenterology, vol. 139, no. 5, pp. 1630.e21641.e2, 2010.

[84] B. C. Miller, Z. Zhao, L. M. Stephenson et al., "The autophagy gene ATG5 plays an essential role in B lymphocyte development," Autophagy, vol. 4, no. 3, pp. 309-314, 2008.

[85] P. Rosenstiel, G. Jacobs, A. Till, and S. Schreiber, "NOD-like receptors: ancient sentinels of the innate immune system," Cellular and Molecular Life Sciences, vol. 65, no. 9, pp. 1361-1377, 2008.

[86] S. J. Ott, M. Musfeldt, D. F. Wenderoth et al., "Reduction in diversity of the colonic mucosa associated bacterial microflora in patients with active inflammatory bowel disease," Gut, vol. 53, no. 5, pp. 685-693, 2004.

[87] J. Qin, R. Li, J. Raes et al., "A human gut microbial gene catalogue established by metagenomic sequencing," Nature, vol. 464, no. 7285, pp. 59-65, 2010.

[88] Z. M. Kanaan, M. R. Eichenberger, S. Ahmad et al., "Clinical predictors of inflammatory bowel disease in a genetically welldefined Caucasian population," Journal of Negative Results in Biomedicine, vol. 11, article 7, 2012.

[89] S. Lesage, H. Zouali, J. P. Cezard et al., "CARD15/NOD2 mutational analysis and genotype-phenotype correlation in 612 patients with inflammatory bowel disease," The American Journal of Human Genetics, vol. 70, no. 4, pp. 845-857, 2002.

[90] T. Petnicki-Ocwieja, T. Hrncir, Y. J. Liu et al., "NOD2 is required for the regulation of commensal microbiota in the intestine," Proceedings of the National Academy of Sciences of the United States of America, vol. 106, no. 37, pp. 15813-15818, 2009.

[91] P. Kuballa, A. Huett, J. D. Rioux, M. J. Daly, and R. J. Xavier, "Impaired autophagy of an intracellular pathogen induced by a Crohn's disease associated ATG16L1 variant," PLOS ONE, vol. 3, no. 10, Article ID e3391, 2008.

[92] Y. G. Kim, N. Kamada, M. H. Shaw et al., “The NOD2 sensor promotes intestinal pathogen eradication via the chemokine CCL2-dependent recruitment of inflammatory monocytes," Immunity, vol. 34, no. 5, pp. 769-780, 2011.

[93] M. Parkes, J. C. Barrett, N. J. Prescott et al., "Sequence variants in the autophagy gene IRGM and multiple other replicating loci contribute to Crohn's disease susceptibility," Nature Genetics, vol. 39, no. 7, pp. 830-832, 2007.

[94] P. Brest, P. Lapaquette, M. Souidi et al., "A synonymous variant in IRGM alters a binding site for miR-196 and causes deregulation of IRGM-dependent xenophagy in Crohn's disease," Nature Genetics, vol. 43, no. 3, pp. 242-245, 2011.
[95] A. Darfeuille-Michaud, C. Neut, N. Barnich et al., "Presence of adherent Escherichia coli strains in ileal mucosa of patients with Crohn's disease," Gastroenterology, vol. 115, no. 6, pp. 1405-1413, 1998.

[96] S. B. Singh, W. Ornatowski, I. Vergne et al., "Human IRGM regulates autophagy and cell-autonomous immunity functions through mitochondria," Nature Cell Biology, vol. 12, no. 12, pp. 1154-1165, 2010.

[97] I. P. Gregoire, C. Richetta, L. Meyniel-Schicklin et al., "IRGM is a common target of RNA viruses that subvert the autophagy network," PLoS Pathogens, vol. 7, no. 12, Article ID e1002422, 2011.

[98] E. Papa, M. Docktor, C. Smillie et al., "Non-invasive mapping of the gastrointestinal microbiota identifies children with inflammatory bowel disease," PLOS ONE, vol. 7, no. 6, Article ID e39242,, 2012.

[99] R. M. Ayling, "New faecal tests in gastroenterology," Annals of Clinical Biochemistry, vol. 49, part 1, pp. 44-54, 2012.

[100] P. F. van Rheenen, E. van de Vijver, and V. Fidler, "Faecal calprotectin for screening of patients with suspected inflammatory bowel disease: diagnostic meta-analysis," British Medical Journal, vol. 341, p. c3369, 2010.

[101] R. Vitali, L. Stronati, A. Negroni et al., "Fecal HMGB1 is a novel marker of intestinal mucosal inflammation in pediatric inflammatory bowel disease," The American Journal of Gastroenterology, vol. 106, no. 11, pp. 2029-2040, 2011.

[102] S. Ehlers, S. H. E. Kaufmann, and Participants of the 99th Dahlem Conference, "Infection, inflammation, and chronic diseases: consequences of a modern lifestyle," Trends in Immunology, vol. 31, no. 5, pp. 184-190, 2010.

[103] A. Marcuzzi, M. Girardelli, A. M. Bianco et al., "Inflammation profile of four early onset Crohn patients," Gene, vol. 493, no. 2, pp. 282-285, 2012.

[104] A. M. Bianco, V. Zanin, M. Girardelli et al., "A common genetic background could explain early-onset Crohn's disease," Medical Hyphoteses, vol. 78, no. 4, pp. 520-522, 2012. 